\title{
Materials and Fuels Complex Human Performance and Nuclear Safety Culture Pocket Guide
}

\section{April 2021}

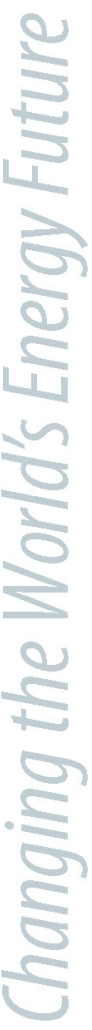

Ronald A Crone

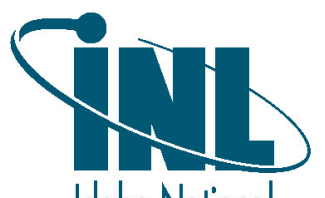

Idaho National

Laboratory 


\section{DISCLAIMER}

This information was prepared as an account of work sponsored by an agency of the U.S. Government. Neither the U.S. Government nor any agency thereof, nor any of their employees, makes any warranty, expressed or implied, or assumes any legal liability or responsibility for the accuracy, completeness, or usefulness, of any information, apparatus, product, or process disclosed, or represents that its use would not infringe privately owned rights. References herein to any specific commercial product, process, or service by trade name, trade mark, manufacturer, or otherwise, does not necessarily constitute or imply its endorsement, recommendation, or favoring by the U.S. Government or any agency thereof. The views and opinions of authors expressed herein do not necessarily state or reflect those of the U.S. Government or any agency thereof. 


\title{
Materials and Fuels Complex Human Performance and Nuclear Safety Culture Pocket Guide
}

\author{
Ronald A Crone
}

April 2021

\begin{abstract}
Idaho National Laboratory Idaho Falls, Idaho 83415
\end{abstract}

http://www.inl.gov

\author{
Prepared for the \\ U.S. Department of Energy \\ Under DOE Idaho Operations Office \\ Contract DE-AC07-05ID14517
}


HBK-104 Rev. 4

\section{Materials and Fuels Complex}

Human Performance and Nuclear Safety Culture Pocket Guide

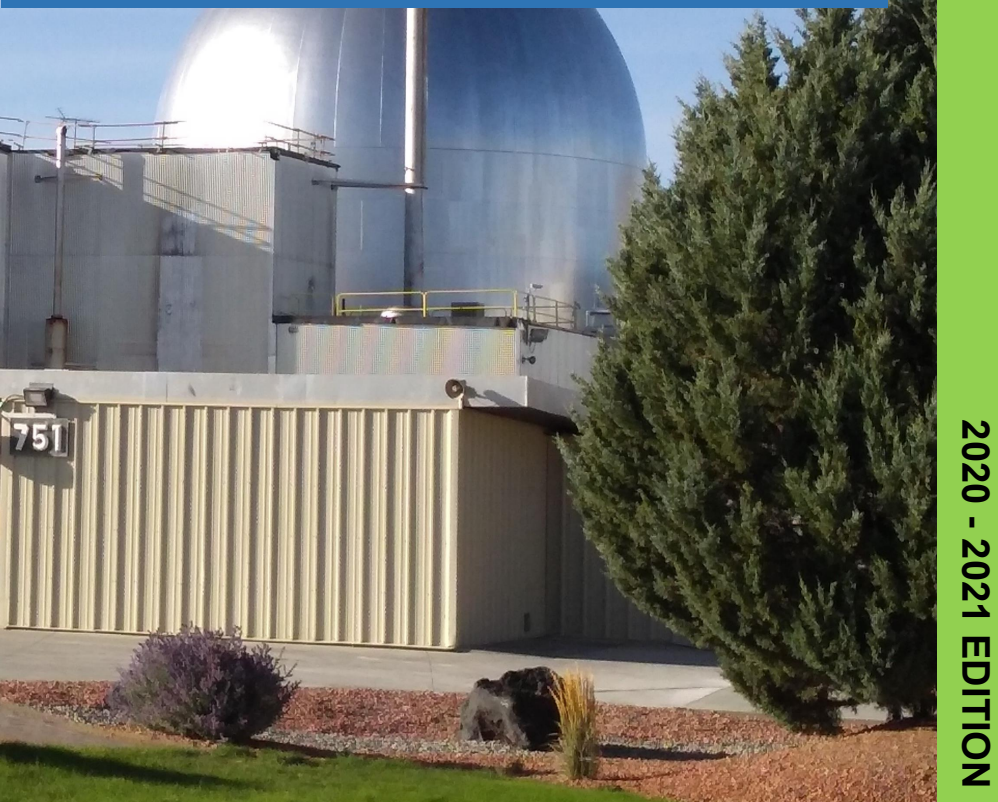




\section{TRUST MODEL - OUR VALUES}

\begin{tabular}{|c|c|}
\hline 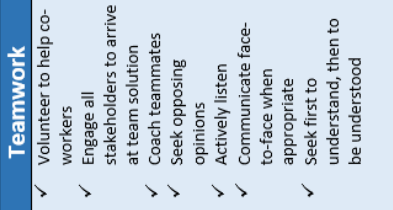 & 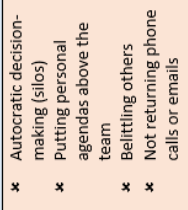 \\
\hline 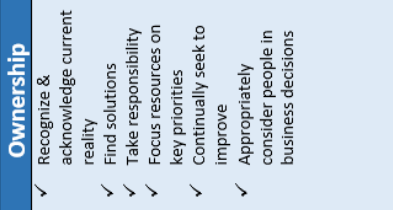 & 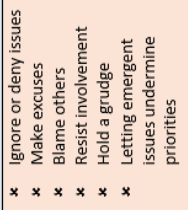 \\
\hline 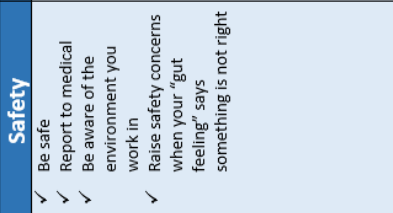 & 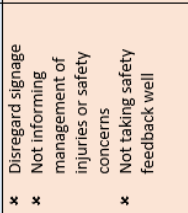 \\
\hline 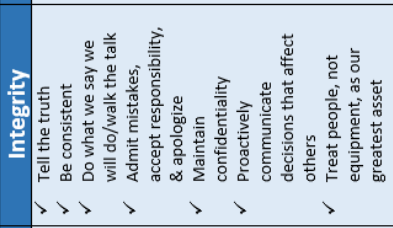 & 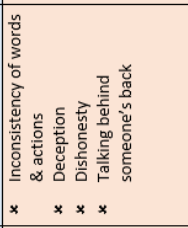 \\
\hline 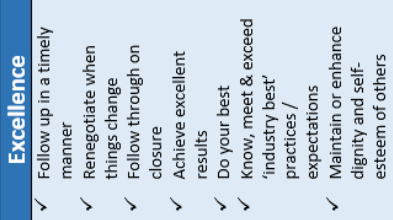 & 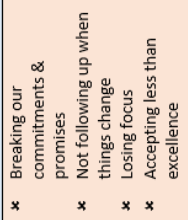 \\
\hline 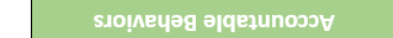 & 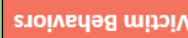 \\
\hline
\end{tabular}




\section{CONTENTS}

FROM THE MFC ASSOCIATE LABORATORY DIRECTOR

Safety Culture 1

Human Performance Improvement 2

Just Culture 2

Professional Development 3

Improvement Agendas 3

FROM THE LABORATORY DIRECTOR 4

CRITICAL SAFETY FUNCTIONS 6

MFC SAFETY CULTURE

Principles for a Strong Safety Culture 7

SAFETY CULTURE PRINCIPLES AND

ATTRIBUTES

8

HUMAN PERFORMANCE ERROR REDUCTION TOOLS

Principles of Human Performance $\quad 19$

Core HPI Tools for All Employees 20

Conditional HPI Tools 21

SITUATIONS WITH HIGH ERROR RATES 22

Error Precursor Table 22

PERFORMANCE MODES 26

IMPORTANT HPI-RELATED TERMS and CONCEPTS 28

MFC WORK CONTROL PROCESS 30

WORK PREPARATION PHASE TOOLS 31

A Questioning Attitude and Your Gut

Feeling 
Pre-Job Brief 32

Take a Minute 34

WORK EXECUTION PHASE TOOLS 36

Self Check/STAR 36

Peer Check 39

Verification Practices 41

Procedure Use and Adherence $\quad 43$

Communication Practices $\quad 45$

Stop When Unsure/Know When to Stop 47

WORK FEEDBACK PHASE TOOLS 49

Post-Job Review 49

MFC Feedback Processes 51

SEVEN RULES TO STAY INJURY FREE 53

LADDER OF ACCOUNTABILITY AND SELF

RESPONSIBILITY

54

IMPORTANT NUMBERS

55

Emergency

55

Bus Operations

55

Clinics

55 


\section{FROM THE MFC ASSOCIATE LABORATORY DIRECTOR}

The facilities at the Materials and Fuels Complex (MFC) contain a diverse collection of nuclear research and development capabilities which enable experiments and engineering to drive the world's nuclear energy future. It is our responsibility to ensure safe, efficient, and reliable operation of MFC facilities to support INL's nuclear science and technology and

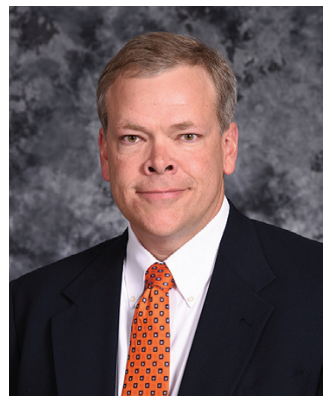
national security missions. The MFC management plan makes it clear that safely achieving this takes the right people using good processes.

\section{Safety Culture}

Because MFC is a nuclear facility complex, unique characteristics and hazards are associated with the nuclear technology employed here. These include radioactive materials contained in spent fuels, irradiated test material, and radioisotopes. In addition, standard industrial hazards are present in the systems, structures, and components which are utilized to perform work at MFC. We must operate, maintain, and perform research at MFC to fulfill our mission without compromising safety to meet schedule pressure or production goals. Having a healthy safety culture ensures that we are committed to safety and are constantly trying to get better by examining our operations and processes to make improvements. 
The safety principles we have adopted are characteristic of an organization that is proactive on safety and respectfully self-critical in seeking to learn, and one which adheres to standards, fosters trust, values employees, and seeks to make prudent decisions. When we each apply these principles in our daily work, all aspects of safety are elevated.

\section{Human Performance Improvement}

The purpose of using human performance improvement (HPl) tools is both to reduce the number of errors that occur and to limit the consequences of these errors. The concepts MFC has adopted accept the fact that all humans are fallible and will make errors. This booklet is intended to provide the fundamental HPI tools and behaviors that can be employed when planning, conducting, and reviewing work.

The values and expectations for the MFC HPI initiative are contained in this booklet and will be reinforced in all aspects of our work and through observations and coaching. Coaching is welcome and expected throughout the entire organization.

\section{Just Culture}

A "just culture" is an environment that allows the organization to answer why something happened so that learning and improvement occurs while preserving relationships and trust. In a just culture:

1. Mistakes are neither punished nor held against an employee who made them while trying to do the right thing.

2. People should not be afraid to make a mistake; they should be afraid of not learning from the ones they do make. 
3. Reporting mistakes, concerns, and events is positive and valued so that the organization can learn, eliminate the error precursors, and improve processes and procedures. This is a key part of having a safety conscious work environment where personnel feel free to raise safety concerns and are encouraged to do so.

4. Organizations must view errors (mental or physical) as an opportunity to learn from and seek to understand the context in which the error was made.

\section{Professional Development}

All MFC staff will engage in professional development opportunities that ensure we are current with the technologies and management systems available to improve our safety and performance. Professional development is key for maintaining our skill set and engaging peers to stimulate thought leadership.

\section{Improvement Agendas}

Each division at MFC has developed an Improvement Agenda which identifies the improvements needed in how we perform our work. The improvement agenda process is a critical self-assessment activity that ensures an opportunity for everyone to identify areas where we can get better. Active participation from all staff is critical for continuous improvement.

Thank you,

Ronald A. Crone, MFC Associate Laboratory Director 


\section{FROM THE LABORATORY DIRECTOR}

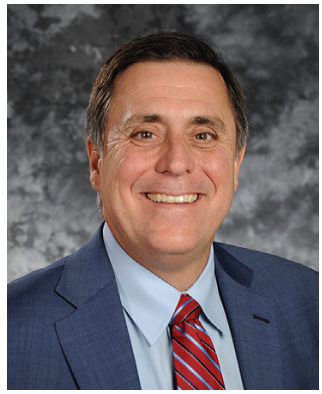

As director of Idaho National Laboratory, I have the privilege of working every day with a talented and dedicated staff that accomplishes great things for its country.

The Materials and Fuels Complex is vital to INL fulfilling its nuclear energy and national security mission. MFC comprises the largest collection of nuclear R\&D facilities in the world. From every corner of the globe, people look to INL, and MFC, to satisfy a growing demand for power in a way that keeps our communities safe, secure, and clean.

That's an awesome responsibility, and one we all take very seriously. But I am proud to tell people that providing the energy that will power our future is not INL's top priority. Its employees are. Making sure our workforce gets home safe and sound every night is my No. 1 concern, and has been since the day I took this job.

INL's vision is for every staff member to have an entire career free from injury, to look out for our co-workers, and to demand the best behavior from ourselves and our colleagues.

Accomplishing our mission and fulfilling our safety vision requires that we establish, maintain, and continually improve upon our safety culture. That requires each of us to comprehend and embrace human performance. 
Human performance is defined simply as "behavior plus results." This means understanding the totality of our thinking and our actions.

At INL, doing things the right way means we never stop thinking about safety. We talk about it with our co-workers, follow guidelines and procedures, and do not hesitate to speak up when we see that something is amiss.

None of us, however, is perfect, and things do happen. When a mistake is made, we report it, honestly and completely, without fear of reprisal or retaliation. I can't stress enough how important this is. Transparency improves us all and makes everyone safer. We use our reporting processes to improve and prevent recurrence. Please continue to report and talk about safety across the complex.

And please continue to put your safety - and that of your colleagues - above all else. Our mission is vital to the future of this nation. We understand that. But we must also understand that our ability to complete this mission is dependent upon each of us embracing a safety culture we have developed over the years as a result of our experiences.

Be aware of your blind spots. Be open to advice and observations from co-workers who might see something you do not and look out for each other. We're all in this together.

Thank you,

Mark Peters, Laboratory Director 


\section{CRITICAL SAFETY FUNCTIONS}

The public and MFC workers are protected from significant radiological exposures in MFC nuclear facilities by compliance with the applicable facility safety bases. INL nuclear facilities and their associated safety bases are documented in LST-715, "INL Nuclear Facilities." Each safety basis identifies a specific set of engineered and administrative controls with their associated safety functions. Representative safety functions include:

- Experimental material behavior is controlled

- Hot cell/glovebox/hood integrity is maintained

- Radioactive material confinement is intact

- Radiation shielding is adequate

- Structural performance is assured under adverse conditions

- Hazard barriers are properly utilized

- Necessary cooling is maintained.

A listing of safety structures, systems, and components (SSCs) for MFC Nuclear Facilities is contained in LST-718, "Safety Systems at INL Nuclear Facilities."

It is of paramount importance that the appropriate operational discipline is applied when working on SSCs. 


\section{MFC SAFETY CULTURE}

\section{Principles for a Strong Safety Culture}

Nuclear and personnel safety are inseparable at MFC. All aspects of safety should be emphasized when working at MFC.

1. Everyone is personally responsible for nuclear and personnel safety

2. Leaders demonstrate commitment to safety

3. Trust permeates the organization

4. Decision-making reflects safety first

5. Nuclear technology is recognized as special and unique

6. A questioning attitude is cultivated

7. Organizational learning is embraced

8. Safety undergoes constant examination. 


\section{SAFETY CULTURE PRINCIPLES AND ATTRIBUTES}

1. Everyone is personally responsible for safety

Responsibility and authority for safety are well defined and clearly understood. Reporting relationships, positional authority, staffing, and financial resources support safety responsibilities. Corporate policies emphasize the overriding importance of safety.

\section{Attributes:}

a. The line of authority and responsibility for safety is defined from the INL Senior Leadership Team (SLT) to the individual contributor. Each of these positions has clearly defined roles, responsibilities, and authorities, designated in writing and understood by the incumbent.

b. Support groups such as human resources, labor relations, and business and financial planning, also understand their roles in contributing to safety.

c. People and their professional capabilities, values, and experiences are regarded as the nuclear organization's most valuable asset. Staffing levels are consistent with the demands related to maintaining safety and reliability.

d. INL SLT members periodically take steps to reinforce safety, including visiting sites to assess management effectiveness first-hand. 
e. The line organization, starting with the lab director, is the primary source of information and the only source of direction. Other parties, such as oversight organizations and committees, review boards, and outside advisors, who provide management information essential to effective self-assessment, are not allowed to dilute or undermine line authority and accountability.

f. All personnel understand the importance of adherence to safety standards. All levels of the organization exercise healthy accountability for shortfalls in meeting standards.

g. The system of rewards and sanctions is aligned with strong safety policies and reinforces the desired behaviors and outcomes.

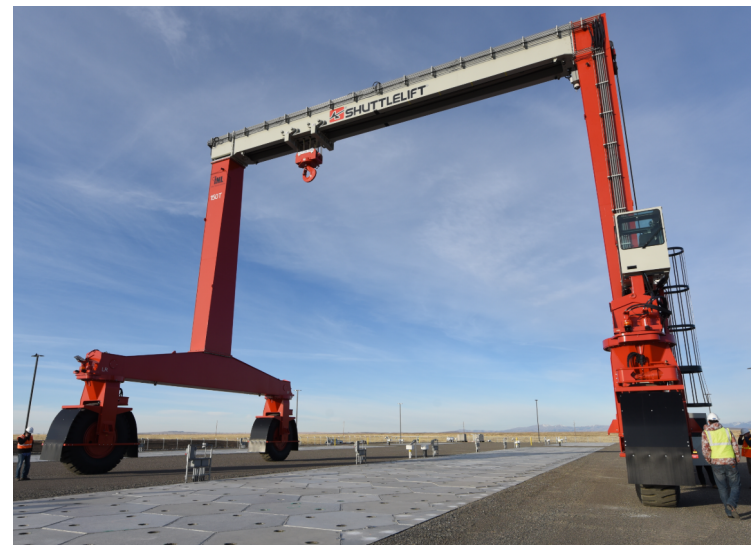




\section{Leaders demonstrate commitment to safety}

Senior managers are the leading advocates of safety and demonstrate their commitment both in word and action. The safety message is communicated frequently and consistently, occasionally as a stand-alone theme. Leaders throughout the organization set an example for safety.

\section{Attributes:}

a. Managers and supervisors practice visible leadership in the field by placing "eyes on the problem," coaching, mentoring, and reinforcing standards. Deviations from expectations are corrected promptly.

b. Management considers the employee perspective in understanding and analyzing issues.

c. Managers and supervisors provide appropriate oversight during safety-significant tests or evolutions.

d. Managers and supervisors are personally involved in high-quality training that consistently reinforces expected worker behaviors.

e. Leaders recognize that production goals, if not properly communicated, can send mixed signals on the importance of safety. They are sensitive to detect and avoid these misunderstandings.

f. The bases, expected outcomes, potential problems, and planned contingencies for important operational decisions are communicated promptly to workers.

g. Selection and evaluation of managers and supervisors consider their abilities to contribute to a strong safety culture. 


\section{Trust permeates the organization}
A high level of trust is established in the organization, fostered, in part, through timely and accurate communication. There is a free flow of information in which issues are raised and addressed. Employees are informed of steps taken in response to their concerns.

\section{Attributes:}

a. People are treated with dignity and respect.

b. Personnel can raise safety concerns without fear of retribution and have confidence their concerns will be addressed.

c. Employees are expected and encouraged to offer innovative ideas to help solve problems.

d. Differing opinions are welcomed and respected. When needed, fair and objective methods are used to resolve conflict and unsettled, differing professional opinions.

e. Supervisors are skilled in responding to employee questions in an open, honest manner. They are recognized as an important part of the management team, crucial to translating safety culture into practical terms.

f. The effects of impending changes (such as those caused by acquisition, bargaining unit contract renegotiations, and economic restructuring) are anticipated and managed such that trust in the organization is maintained. 
g. Complete, accurate, and forthright information is provided to oversight, audit, and regulatory organizations.

h. Managers regularly communicate to the workforce important decisions and their bases, as a way of building trust and reinforcing a healthy safety culture. Worker understanding is periodically checked.

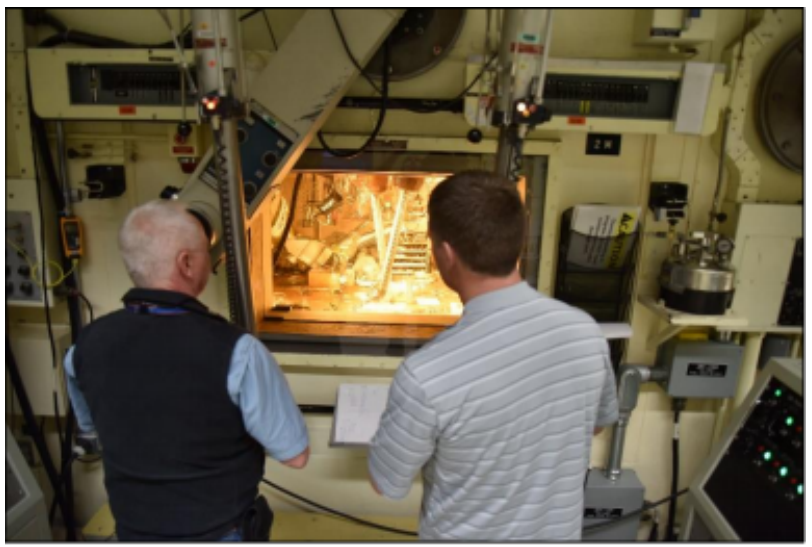




\section{Decision making reflects safety first}

Personnel are systematic and rigorous in making decisions that support safe, reliable laboratory operation. Operators, technicians, and researchers are vested with the authority and understand the expectation, when faced with unexpected or uncertain conditions, to place the equipment in a safe condition. Senior leaders support and reinforce conservative decisions.

\section{Attributes:}

a. The organization maintains a knowledgeable workforce to support a broad spectrum of operational and technical decisions. Outside expertise is employed when necessary.

b. Managers, supervisors, and staff clearly understand and respect each other's roles in decision-making.

c. Lab personnel apply a rigorous approach to problem solving. Conservative actions are taken when understanding is incomplete.

d. Single-point accountability is maintained for important safety decisions, allowing for ongoing assessment and feedback as circumstances unfold.

e. Candid dialogue and debate are encouraged when safety issues are being evaluated. Robust discussion and healthy conflict are recognized as a natural result of diversity of expertise and experience.

f. Decision-making practices reflect the ability to distinguish between "allowable" choices and prudent choices. 
g. When previous operational decisions are called into question by new facts, the decisions and associated underlying assumptions are reviewed to improve the quality of future decisions.

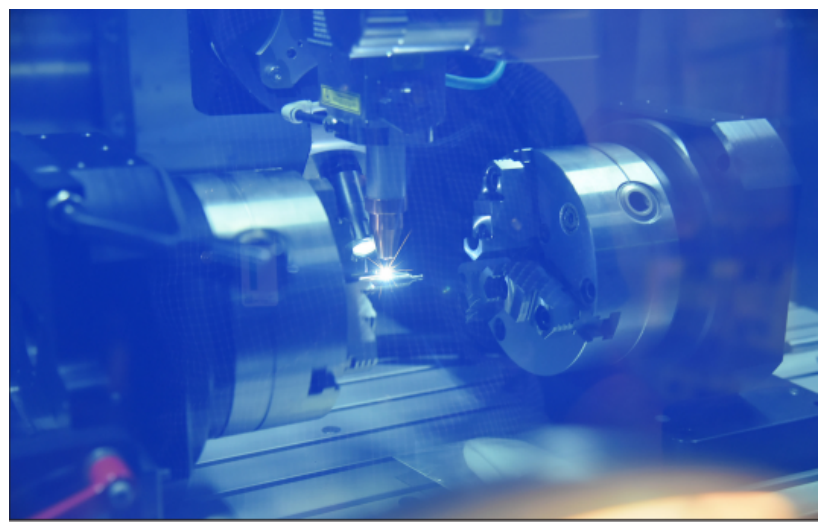




\section{Nuclear technology is recognized as special and unique}

The special characteristics of nuclear technology are considered in all decisions and actions. Reactivity control/criticality and radioactive material handling and confinement are valued as essential, distinguishing attributes of the nuclear work environment.

\section{Attributes:}

a. Activities that could affect criticality are conducted with particular care and caution.

b. Features designed to maintain critical safety functions are recognized as particularly important.

c. Design and operating margins are carefully guarded and are changed only with great thought and care. Special attention is placed on maintaining radioactive material handling and containment barriers and defense in depth.

d. Equipment is meticulously maintained well within design requirements.

e. Research activities are governed by comprehensive, high-quality processes and procedures.

f. Employee mastery of nuclear research facility fundamentals, as appropriate to the job position, establishes a solid foundation for sound decisions and behaviors. 


\section{A questioning attitude is cultivated}

Individuals demonstrate a questioning attitude by challenging assumptions, investigating anomalies, and considering potential adverse consequences of planned actions. This attitude is shaped by an understanding that accidents often result from a series of decisions and actions that reflect flaws in the shared assumptions, values, and beliefs of the organization. All employees are watchful for conditions or activities that can have an undesirable effect on safety.

\section{Attributes:}

a. While individuals expect successful outcomes of daily activities, they recognize the possibility of mistakes and worst-case scenarios. Contingencies are developed to deal with these possibilities.

b. Anomalies are recognized, thoroughly investigated, promptly mitigated, and periodically analyzed in the aggregate.

c. Personnel do not proceed in the face of uncertainty.

d. Workers identify conditions or behaviors that have the potential to degrade operating or design margins.

Such circumstances are promptly identified and resolved.

e. Employees understand that complex technologies can fail in unpredictable ways. They are aware that latent problems can exist, and they make conservative decisions considering this potential.

f. Groupthink is avoided through diversity of thought and intellectual curiosity. Opposing views are encouraged and considered. 


\section{Organizational learning is embraced}

Operating experience is highly valued, and the capacity to learn from experience is well developed. Training, self-assessments, corrective actions, and benchmarking are used to stimulate learning and improve performance.

\section{Attributes:}

a. The organization avoids complacency and cultivates a continuous learning environment. The attitude that "it can happen here" is encouraged.

b. Training upholds management standards and expectations. Beyond teaching knowledge and skills, trainers are adept at instilling safety values and beliefs.

c. Individuals are well informed of the underlying lessons learned from significant events, and they are committed to not repeating these mistakes.

d. Expertise in root cause analysis is applied effectively to identify and correct the fundamental causes of events.

e. Processes are established to identify and resolve latent organizational weaknesses that can aggravate relatively minor events if not corrected.

f. Employees have confidence that issues with significant safety implications are prioritized, tracked, and resolved in a timely manner. 


\section{Safety undergoes constant examination}

Oversight is used to strengthen safety and improve performance. Safety is kept under constant scrutiny through a variety of monitoring techniques, some of which provide an independent "fresh look."

\section{Attributes:}

a. A mix of self-assessment and independent oversight reflects an integrated and balanced approach. This balance is periodically reviewed and adjusted as needed.

b. Periodic safety culture assessments are conducted and used as a basis for improvement.

c. The pitfalls of focusing on a narrow set of performance indicators are recognized. The organization is alert to detect and respond to indicators that may signal declining performance.

d. The insights and fresh perspectives provided by quality assurance, assessment, employee concerns, and independent oversight personnel are valued. 


\section{HUMAN PERFORMANCE ERROR REDUCTION TOOLS}

\section{Principles of Human Performance}

People are fallible; even the best make mistakes.

Error-likely situations are predictable, manageable, and preventable.

Individual behavior is influenced by organizational processes and values.

People achieve high levels of performance based largely on the encouragement and reinforcement received from leaders, peers, and subordinates.

Events can be avoided by an understanding of the reasons mistakes occur and through the application of lessons learned from past events.

HPI tools assist us in realizing this goal. There are Core HPI tools for all employees to know and use in our jobs, and there are Conditional HPI tools that are more group or job-specific. All Essential and specific key Conditional tools are presented individually on the following pages with the phase of work (preparation, execution, feedback) where they are most likely to apply.

Having a booklet and a set of HPI tools does not guarantee error-free work and event-free operation. It takes each of us being prepared, mentally engaged, having a questioning attitude, good communication, and correctly implementing the practices discussed here. 


\section{Core HPI Tools for All Employees}

All employees should understand HPI Fundamentals and how the following error-reduction tools apply to what they do:

\section{Questioning Attitude}

\section{Pre-Job Brief}

Take a Minute

\section{Stop When Unsure}

\section{Peer Check/Peer Review}

\section{Self Check using STAR}

\section{Effective Communication Practices}

\section{Procedure Use and Adherence}




\section{Conditional HPI Tools}

The following tools apply to specific job situations, whether physical work or technical work. Important conditional tools are highlighted in red.

\begin{tabular}{l|c|c|c|c|c}
\cline { 2 - 6 } & \multicolumn{2}{|c|}{ Type of work } & \multicolumn{3}{c}{ Phase of the Job } \\
\cline { 2 - 6 } & Physical & Technical & Preparation & Execution & Feedback \\
\hline Decision-Making & $\bullet$ & $\bullet$ & $\bullet$ & $\bullet$ & \\
\hline Do Not Disturb & & $\bullet$ & $\bullet$ & $\bullet$ & \\
\hline Flagging & $\bullet$ & & & $\bullet$ & \\
\hline Place-Keeping & $\bullet$ & & & $\bullet$ & \\
\hline Phonetic Alphabet & $\bullet$ & $\bullet$ & $\bullet$ & $\bullet$ & $\bullet$ \\
\hline Post-Job Review & $\bullet$ & $\bullet$ & & & $\bullet$ \\
\hline Pre-Job Brief & $\bullet$ & $\bullet$ & $\bullet$ & & \\
\hline Project Planning & $\bullet$ & $\bullet$ & $\bullet$ & & \\
\hline Signature & $\bullet$ & $\bullet$ & $\bullet$ & $\bullet$ & $\bullet$ \\
\hline $\begin{array}{l}\text { Task Preview } \\
\text { Three-Way } \\
\text { Communication }\end{array}$ & $\bullet$ & & $\bullet$ & & \\
\hline Turnover & $\bullet$ & $\bullet$ & $\bullet$ & $\bullet$ & \\
\hline $\begin{array}{l}\text { Validate } \\
\text { Assumptions }\end{array}$ & $\bullet$ & $\bullet$ & $\bullet$ & $\bullet$ & \\
\hline $\begin{array}{l}\text { Vendor Oversight } \\
\text { Verification- } \\
\text { Concurrent (CV) }\end{array}$ & $\bullet$ & $\bullet$ & $\bullet$ & $\bullet$ & \\
\hline $\begin{array}{l}\text { Verification - } \\
\text { Independent (IV) }\end{array}$ & $\bullet$ & $\bullet$ & & $\bullet$ & \\
\hline
\end{tabular}




\section{SITUATIONS WITH HIGH ERROR RATES}

\section{Error Precursor Table}

\section{Task Demands}

Time pressure (in a hurry)

High workload (memory requirements)

Simultaneous, multiple tasks

Repetitive actions, monotonous

Irrecoverable acts

Interpretation requirements

Unclear goals, roles, and responsibilities

Lack of or unclear standards

\section{Work Environment}

Distractions/interruptions

Changes/departures from routine

Confusing displays or controls

Workarounds/out of service instruments

Hidden system response Unexpected equipment

\section{Individual Capabilities}

Unfamiliarity $\mathrm{w} /$ task/first time

Lack of knowledge (mental model)

New technique not used before

Imprecise communication habits

Lack of proficiency/inexperience Indistinct problem solving skills

Hazardous attitude for critical task Illness/fatigue

\section{Human Nature}

Stress (limits attention)

Habit patterns

Assumptions (inaccurate mental picture)

Complacency/overconfiden ce

Mindset ("tuned" to see)

Inaccurate risk perception 
Unexpected equipment conditions

Lack of alternative indication

Personality conflicts
Inaccurate risk perception (Pollyanna)

Mental shortcuts (biases)

Limited short-term memory 


\section{PERFORMANCE MODES}

HPI recognizes that each of us makes errors, and it seeks to minimize the frequency and reduce the consequences of errors made during work at MFC.

As illustrated below, tasks are performed in one of three basic modes depending on task familiarity and attention requirements. We can go in and out of all three depending on our awareness, knowledge, and available resources.

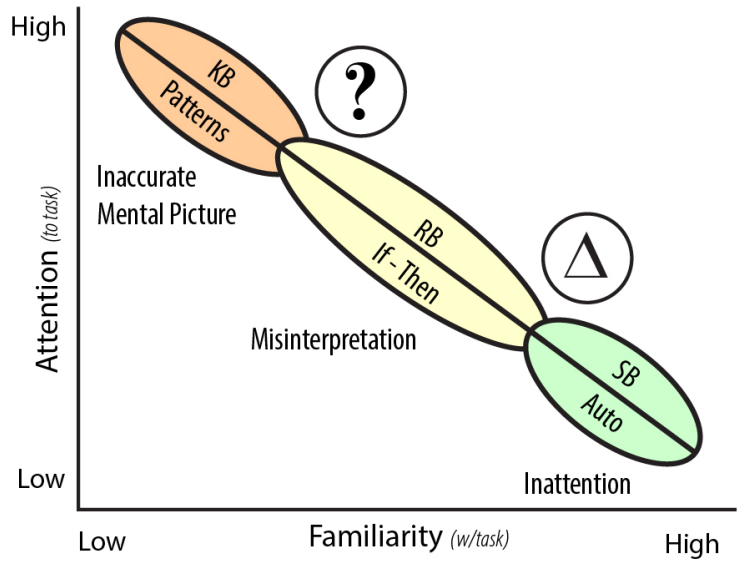

Being in knowledge-based performance (KB) mode is normally infrequent; however, there are components of many experiments that, by their nature, occur in knowledgebased performance mode. The need for relevant information is paramount, and attention must become more focused, e.g., when troubleshooting, resolving conflicting indications, performing root cause analyses or engineering calculations. An inaccurate mental model is the prevalent error mode. 
Fifteen percent of errors occur here. The error rate is $\sim 1$ in 2. Communication, problem-solving, a questioning attitude, and team skills are key to avoiding error in KB mode.

Rule-based performance (RB) mode is governed by memorized or written rules to improve the worker's interpretation of the work situation so that the right response is selected and used. Procedures are predetermined solutions to possible work situations that require specific responses. Sixty percent of errors occur here. Misinterpretation is the primary error mode. Error rate is $\sim 1$ in 1,000 . Effective tools to reduce error are procedure adherence, pre-job briefings, and a questioning attitude.

Skill-based performance (SB) mode involves highly practiced and familiar tasks that can be done with little conscious thought (e.g., shaving, driving in normal conditions). Twenty-five percent of errors occur here, and inattention is the primary error mode. Error rate is $\sim 1$ in 10,000 actions. Use self-checking to reduce errors.

The chance for error increases when people make choices or decisions in the field. Properly transitioning between performance modes requires situational awareness and a questioning attitude to recognize evolving work situations. If tools, procedures, or knowledge is inadequate, stop and address the issue.

For additional information, refer to the DOE Human Performance Handbook, Vol. 1, Chapter 2. 


\section{IMPORTANT HPI-RELATED TERMS and CONCEPTS}

Critical Step: A procedure step or action that is within the control of the worker(s), typically irreversible, and when performed incorrectly could result in an unwanted outcome.

Defenses: Proactive measures to reduce the frequency and severity of errors and events. Controls guide, coordinate, or regulate performance and discourage unsafe acts. Barriers reduce severity of consequences.

Drift: Gradual deviation from espoused standards of performance. The difference between work as perceived and work as performed.

Event: An unwanted and undesirable change in the state of facility structures, systems, or components, human and organizational conditions (health, behavior, administrative controls, environment, etc.) that exceed established criteria.

Eyes on Path: A vigilant cautiousness for potential hazards in the path of ingress or egress (i.e., watching for slip, trip, or fall hazards in your pathway, etc.).

Failing Safely: Understanding the critical steps, expecting failure at the critical step, and identifying the defenses in place or needed to prevent/mitigate an unwanted outcome.

Feedback: Information about past or present behavior and results that gives an individual or the organization the opportunity to change and improve. Feedback is key to the organizational and individual learning process.

Hazard: Anything that poses a threat to the health and well-being of people or to the mission of the organization. 


\section{Heightened Awareness at Critical Steps: Increased}

awareness of the potential human errors and their consequences related to the performance of critical steps and what $\mathrm{HPI}$ tools are appropriate to use.

Human Error: The failure of planned actions to achieve their desired results. There are two types of error:

- Active errors are observable physical actions that change equipment, system, or plant state, resulting in immediate undesired consequences.

- Latent errors include actions, directives, and decisions that either create the conditions for a later error or fail to prevent the effects of error.

Latent Organizational Weakness: Undetected deficiencies in processes that create error likely situations.

Positive Control: Active measure(s) to ensure that what is intended to happen is what happens and that is all that happens.

Safety Culture: The organization's values and beliefs-modeled by its leaders and internalized by its members-

that serve to make safety the overriding priority.

Situational Awareness: The accuracy of a person's current knowledge and understanding of working conditions compared to actual conditions at a given time.

Unsafe Attitude: Unhealthy beliefs and assumptions about workplace hazards that blind people to the pre-existing factors that make human error, personal injury, or physical damage to equipment more likely.

Willful Violation: Deliberate or erroneous deviation from safe operating procedures, standards, or rules. 


\section{MFC WORK CONTROL PROCESS}

MFC work control processes are governed by various operating procedures, procedures directing knowledge-based work, and INL's Integrated Safety Management System (ISMS).

Work planning and control processes derived from ISMS are key opportunities for enhancement by application of HPI concepts and tools. Human performance has an impact at each stage of the ISMS work cycle.

Three phases to the work execution process are preparation, execution, and feedback.

Preparation. Anticipate possible active errors and their consequences and incorporate appropriate defenses, especially at critical steps: walkdowns-identify potential jobsite challenges to error-free performance; task assignment-put the right people on the job in light of the job's task demands. ISMS core functions 1, 2, and 3.

Execution. Perform work with a heightened awareness at critical steps, maintain situation awareness; use human performance tools rigorously for important human actions; avoid unsafe or at-risk work practices; and support execution with quality supervision and teamwork. Positive Control is applied at critical steps to ensure that WHAT IS INTENDED TO HAPPEN IS WHAT HAPPENS AND THAT IS ALL THAT HAPPENS. ISMS core function 4.

Feedback. Report and learn by conveying information on how the job went/was performed, on the quality of work preparation, related resources, and workplace conditions through observations performed by managers and supervisors. ISMS core function 5 . 


\section{WORK PREPARATION PHASE TOOLS}

\section{A Questioning Attitude and Your Gut Feeling}

An active questioning attitude is important to all phases and all types of work at MFC. Everyone is expected to challenge assumptions, investigate anomalies, and consider the potential adverse consequences of unplanned actions. Be wary of any unforeseen external influence and have a heightened awareness at critical steps. A questioning attitude is an underlying behavior common to virtually all HPI tools, requiring flexible thinking during each task to respond properly to "gut feelings."

- During work preparation, a questioning attitude fosters awareness of uncertainties, assumptions, risks, error precursors, and the significance of a decision or action before proceeding.

- During work execution, a questioning attitude is part of alerting people to potential hazards, warning signs, critical steps, error-likely situations, procedural inadequacies, and other uncertainties in the work environment. The correct response is to stop and resolve the issue.

- During the work feedback phase, it brings to mind those items recognized during execution as lessons learned and input to process improvement.

Bottom line: If your gut tells you something you or your co-worker is about to do is not right, pause and employ the appropriate human performance tool before proceeding. If someone questions you, help them resolve their gut feeling. 


\section{Pre-Job Brief}

\section{Why?}

To ensure all participants know the objectives and accept their roles in achieving them

To identify and communicate the critical steps, if any

To communicate expected hazards and controls

To discuss potential error traps

To understand what to do when things go wrong.

\section{When?}

Prior to beginning a task or major phase for everyone on the job or after a prolonged break.

\section{How?}

Conduct a pre-job brief in accordance with LWP-9201, "Briefings." A goal of the pre-job brief is active dialogue among participants that helps staff to "see" error-likely situations, especially at critical steps or phases of the activity. This prepares staff for the desired accomplishments and helps them know what to avoid. Follow a graded approach for the brief based on the complexity, frequency, and risk of the job. Do the following with a questioning attitude:

- Review the scope of work (ISMS Core Function \#1) focusing on the objective, critical steps, and required resources

- Discuss previous relevant operating experience

- Review the potential hazards and problems (ISMS Core Function \#2)

- Review appropriate controls and barriers (ISMS Core 
Function \#3). Develop contingency plans per the appropriate procedure

- Perform the work (ISMS Core Function \#4) using the Take a Minute tool as appropriate prior to work start.

Knowledge-based technical task teams can implement this tool as well for safety and technical safety requirement (TSR)-related document changes and calculations.

\section{At-risk practices to avoid:}

- Thinking the job is "routine" or "simple"

- Failing to cover the scope of the task and critical steps

- Gathering signatures prior to brief completion

- Going through the motions of a pre-job brief, unengaged attendees, and assumption of roles and responsibilities. 


\section{Take a Minute}

\section{Why?}

A quick jobsite walk-around and look-around are used to verify that jobsite conditions and all other assumptions made at the pre-job briefing are correct, to establish clear situational awareness, and to verify each worker is ready to proceed. It promotes FOCUS, situational awareness, readiness, and hazard recognition.

\section{When?}

When first arriving at or returning to the jobsite, take the time to get (re)acquainted with the immediate work area.

\section{How?}

Explore the jobsite during the FIRST few minutes by walking and looking around at the work area and adjacent surroundings to identify and consider conditions such as:

- Industrial safety, radiological, and environmental hazards (and required PPE)

- Sensitive equipment in the area

- Electronic dosimeter readings if in an RCA

- Critical indicators for task success

- Error precursors (at critical steps)

- Work area conditions inconsistent with those listed in the procedure or discussed during the pre-job briefing

- Problems, barriers, contingencies.

Have a questioning attitude for unexpected conditions and task demands. 


\section{At-risk practices to avoid:}

- Hurrying

- Thinking the job is "routine" or "simple"

- Believing nothing bad can happen

- Failing to discuss precautions with co-workers

- Remaining silent when you have a "gut feeling"

- Proceeding when unsure.

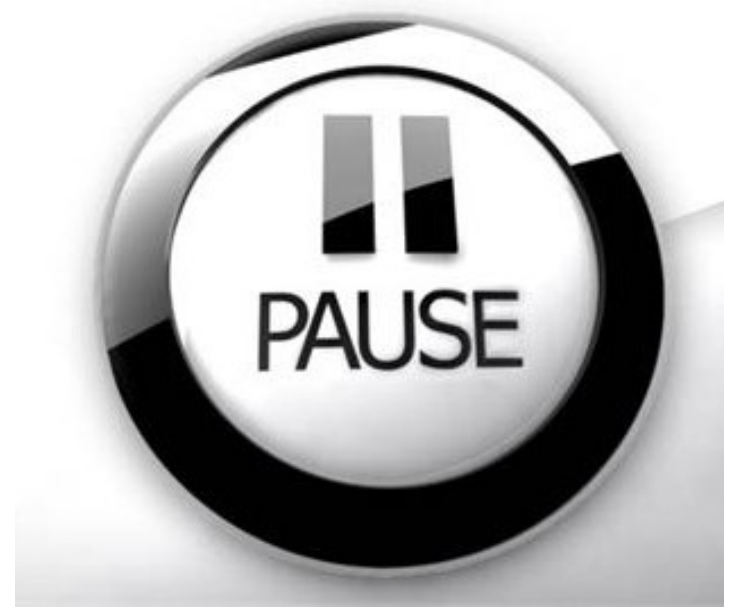




\section{WORK EXECUTION PHASE TOOLS}

\section{Self Check/STAR}

\section{Why?}

To ensure you take the correct action on the intended component BEFORE the action is taken and to focus attention on the details of the task and ensure understanding.

\section{When?}

STAR is performed when a person self-identifies that there is a reasonable potential that an action could be done incorrectly or inappropriately and have an undesirable consequence, or whenever a worker is uncomfortable with a situation. When to use STAR:

- Prior to critical tasks

- Following task interruption

- Prior to any equipment manipulation

- When time pressures exist

- Prior to performing an unfamiliar or first-time task

- When a gut-feeling indicates something is not right.

\section{How?}

1. Stop-Pause before performing the operation or manipulation, especially at critical steps, decision points, or touch points. Eliminate distractions. 
2. Think-Focus attention on the step to be performed. Verify the action is appropriate for equipment or system status. Anticipate expected results of the action and their indications. Consider what actions to take should an unexpected result occur (that is, create contingency plans).

When going through the Think step, ask yourself the following questions:

$\square$ What do I want to get done?

$\square \quad$ What could go wrong?

$\square \quad$ Am I ready for anything that goes wrong?

$\square$ Does my work control cover what goes right or wrong?

$\square \quad$ What am I going to check when I am done? 


\section{Act-}

- Without losing eye contact, touch the component, label, etc.

- Compare component, label, etc., with the directing document

- State the component name or ID aloud (without distracting others)

- Without losing physical contact established earlier, perform the action.

4. Review-Verify anticipated result obtained. Perform contingency tasks if expected result does not occur.

\section{At-risk practices to avoid:}

- Being distracted and losing visual or physical contact

- Talking on the telephone or conversing with another person during a manipulation or critical action

- Self-checking without the work control document

- Attempting to perform more than one action at a time

- Continuing with the action when questions or discrepancies occur. 


\section{Peer Check}

\section{Why?}

To prevent an error by the task performer.

\section{When?}

Perform peer checks whenever:

- A person self identifies a situation where help from a co-worker will reduce the likelihood of an error or unintended consequence.

\section{Examples:}

- Previous experience includes an error

- Directed by the task leader in the pre-job brief

- Prior to transmitting an important response or report

- Ensuring operation of the right equipment

- Executing the correct procedure or process step

- Mitigating an error-likely environment.

\section{How?}

Two people (performer and peer) self-checking in parallel, agreeing together that the action is the correct action to perform on the correct component. This technique takes advantage of a fresh set of eyes not trapped by the task-focused mind-set of the performer; a peer may see hazards the performer does not see.

The peer observes the performer before and during execution to check that the performer takes the correct action on the correct component. If the performer's action is consistent with the intended action, the peer informs the performer that the action taken is correct. 
The STAR technique can be jointly used by performer and peer to complete the peer check.

\section{At-risk practices to avoid:}

- Peer inattentiveness

- Poor communication

- Failing to use a questioning attitude to ensure thoroughness and safety

- Failing to conduct a peer review prior to sending important email responses, presentations, analyses, and reports to transmission or submission. 


\section{Verification Practices}

\section{Why?}

In MFC's various facilities, this step confirms that components such as valves, switches, and circuit breakers are correctly positioned. In knowledge work, it is used to verify assumptions, standards, calculations, and design via checking and reviewing.

\section{When?}

Concurrent Verification (CV): A method of checking an operation, an act of positioning, or a calculation, in which the verifier independently observes and/or confirms the operation or calculation.

Independent Verification (IV): The act of checking, by a separate qualified person, that a given operation or component position conforms to established criteria. Independent verifiers are physically separated by time and distance so as not to influence the other. Verification only checks for conformance with the criteria; it does not alter the status of the equipment or the position of components.

\section{How?}

The correct component is identified and its position/status is verified without subjecting the verifier to undue hazard exposure.

CV: Two people working together at the same time and place separately confirm action correctness before, during, and after an action. This is usually documented with the signatures or initials of the performer and verifier in the guiding document. The expected outcome is also verified. 
IV: Two individuals working independently (separated by both time and distance) confirm the position or condition of a component after the original act that placed it in that condition, documenting the position or condition with the signatures or initials of the performer and verifier in the guiding document.

\section{Always}

- Use the verification technique called for

- Establish verifier and performer roles beforehand

- Discuss the hazards surrounding the act to be verified.

\section{At-risk practices to avoid:}

- Disregarding self-check through the verification process

- Failure to communicate roles and intent

- Using unqualified people to perform CV or IV. 


\section{Procedure Use and Adherence}

\section{Why?}

Procedure adherence means understanding the procedure's intent and purpose and following its direction to ensure the desired outcome is achieved safely and reliably. Users should follow procedures, mindful of the impact their actions could have on the facility or equipment before work execution.

\section{When?}

Whenever:

- A procedure exists for a work activity

- Required by TSRs or other technical documents.

\section{How?}

The user performs all actions as written in the sequence specified unless they cannot be performed safely and correctly. When this cannot be done or if the procedure leaves you unsure, stop the activity and get guidance. Revise the procedure if necessary.

- Operations per LWP-9600, "Conduct of Operations for the Idaho National Laboratory," MCP-9600, "Conduct of Operations for Materials and Fuels Complex Facility Operations," and LWP-6200, "Maintenance Integrated Work Control Process"

- Verify use of the latest revision

- Read the procedure, know precautions and limitations, and fulfill prerequisites prior to work start

- Sign, initial, or check off steps as directed

- Use the procedure according to its designed level of use or as directed. 


\section{At-risk practices to avoid:}

- Failure to review a procedure prior to the job

- Using previous procedure revision or missing relevant sections

- Skipping steps because they have been unnecessary before

- Performing a procedure without knowing the critical steps

- Checking-out and mindlessly following a procedure

- Avoiding (giving or receiving) feedback on the technical accuracy and usability (or lack thereof). 


\section{Communication Practices}

\section{Why?}

Clear, concise, complete, and consistent communication is essential to effective teamwork, transfer of critical information, and use of some error-prevention methods such as peerchecking, verification, coaching, and pre- and post-job briefs.

\section{When?}

Good communication practices are always appropriate, and particularly necessary:

- In answering operations and maintenance-related questions

- When performing equipment manipulation

- In discussing the scope or review of a proposed change

- At the end of a meeting to summarize and ensure understanding

- When directing actions during emergency operations.

\section{How?}

The four goals in any communication are: (1) to have the message received; (2) to have the message understood;

(3) to initiate action; (4) to maintain or enhance the relationship. The following can help achieve those goals.

- Three-way communication

- Phonetic alphabet

- Use of commonly known acronyms only. 


\section{At-risk practices to avoid:}

- Assuming your message has been received

- Improper use of communication methods.

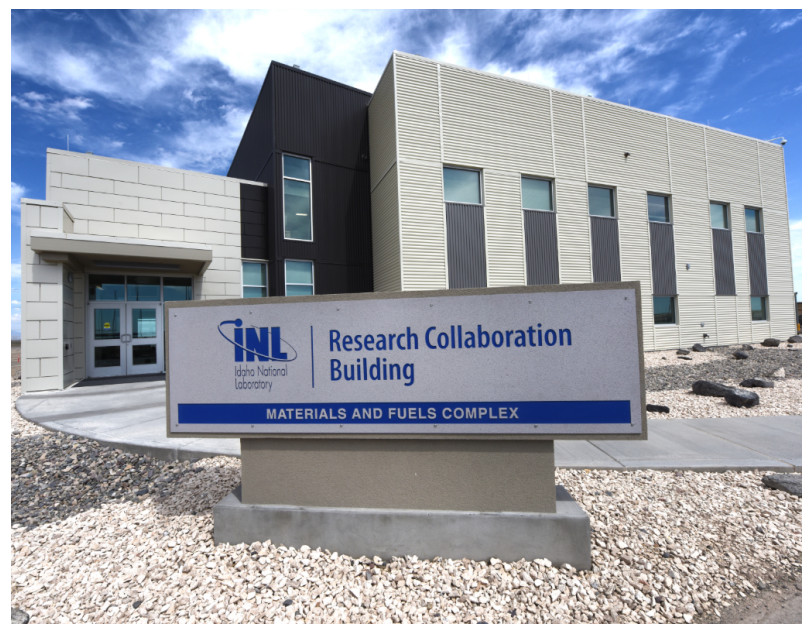




\section{Stop When Unsure/Know When to Stop}

\section{Why?}

Suspend work when it is perceived that a situation exists that will place you or others in an unsafe situation, may have an adverse impact on the environment, or could have observed undesirable trends related to operations, safety, the environment, quality, or security (see LWP-14002, "Timeout and Stop Work Authority"). Stop work:

- To avoid errors when uncertain or unsafe conditions are identified

- To prompt teams or individuals to get help if conditions change or are inconsistent with expectations

- To prevent release of or exposure to a known hazard.

\section{When?}

Suspend work when the task or activity is not in compliance with established procedures or the pre-job brief, conditions change, or unexpected results are obtained. Also when:

- You are unsure or unclear on how to proceed

- You recognize unsafe conditions

- You notice plant or task conditions change

- Your results are inconsistent with your expectations.

\section{How?}

- Suspend the work activity

- Warn others who may be at risk

- If not in imminent danger, review the situation or condition, implement changes, inform coworkers, and resume work 
- Notify the on-duty shift supervisor and task leader if required per ISMS

- If the situation cannot be resolved, issue a Stop Work Order.

At-risk practices to avoid:

Proceeding in the face of uncertainty, unsafe or unexpected conditions, or imminent danger.

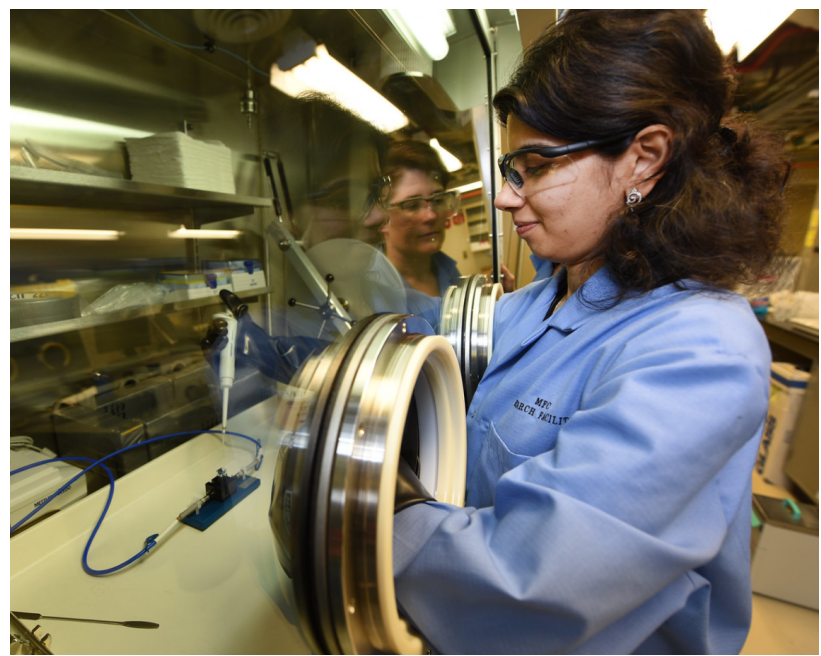




\section{WORK FEEDBACK PHASE TOOLS}

\section{Post-Job Review}

\section{Why?}

Post-job reviews provide an opportunity:

- To improve processes and practices and prevent future errors and events

- To protect workers on future tasks

- To eliminate causes of errors

- To improve safety, efficiency, and reliability

- To improve the process and the work environment.

\section{When?}

Perform a post-job review immediately after a job at a safe, pre-determined location. Conduct a post-job brief as determined by the task leader, shift supervisor, or management if any of the following occur:

- The expected results were not obtained

- The job should be done differently in the future

- A procedure could not be completed verbatim

- Training was not appropriate or was insufficient

- The work process was less than effective

- An event, equipment damage, or injury occurred. 


\section{How?}

For Operations and Maintenance, use LWP-9201 to guide the review. Convene as many task participants as possible to review and document:

- Problems encountered

- Recommendations for improvements

- Corrective actions taken to resolve problems

- Safety issues encountered.

Technical task teams can implement this tool, as well, for safety and TSR-related document changes, calculations, or to gather lessons learned from experiments.

\section{At-risk practices to avoid:}

- Failing to conduct a post-job review when one would have benefited future work

- Inadequate changes necessary to improve the process given the post-job review results. 


\section{MFC Feedback Processes}

\section{Why?}

Follow feedback processes to ensure that concerns about processes and equipment are recognized by management for prioritization and attention. Reporting and feedback are important methods to sustain a learning organization. Report any concern that could impact personnel or plant safety, efficiency, or reliability.

\section{When?}

If there is imminent risk of compromising safety, report to the shift supervisor, plant manager, maintenance manager, or nuclear safety manager as soon as possible and document the discussion afterwards.

\section{How?}

Provide feedback for process improvement and report other concerns as soon as convenient, using proper methods:

- Report emerging conditions directly to your supervisor

- Report ideas for improvement or concerns regarding a specific plant activity to the shift supervisor

- Participate in post-job reviews (formal meeting or documentation)

- Provide performance assurance with lessons learned to be uploaded to the OPEXShare database

- Initiate a request for modification to improve plant systems, structures, or components 
- Document observations on the MFC observation checklist

- Initiate a non-conformance report for defective or degraded materials.

\section{At-risk practices to avoid:}

- Rationalizing to yourself on why you shouldn't provide feedback or report your concern

- Disregarding the question "how could it have been done better?" 


\section{SEVEN RULES TO STAY INJURY FREE}

1. Pay attention to your surroundings when traveling between locations and between different walking surfaces

2. Always wear the appropriate Personal Protective Equipment (PPE) for the work activities being performed. Contact Industrial Safety or Industrial Hygiene to assist with determining the type of PPE needed.

3. Use proper lifting techniques and never lift heavy or bulky objects by yourself

4. Have an ergonomic evaluation of your work area and always take regular breaks when performing repetitive tasks

5. If you must strain in any way to perform a task, get help before proceeding

6. Pause work when conditions change or when the task is different from that expected in order to identify new hazards and controls or seek further direction from the proper authority

7. Drive like your kids are in your car or that your kids are running through the MFC parking lot. 


\section{LADDER OF ACCOUNTABILITY AND SELF RESPONSIBILITY}

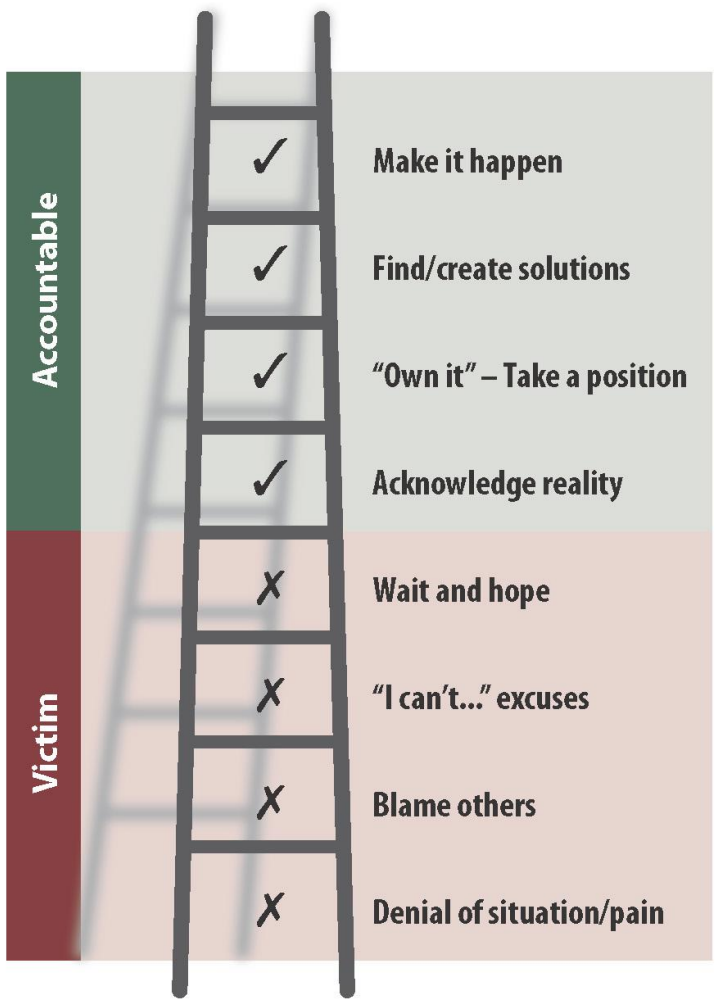




\section{IMPORTANT NUMBERS}

\section{Emergency}

For ambulance, police, or fire first call the following numbers:

Idaho Falls and Site location - From an office phone, dial 911

Warning Communications Center (WCC) 208-526-1515

Fire Alarm Center - Alternative emergency number for on-site events 208-526-2213

\section{Bus Operations}

To learn about severe road conditions and bus routes, call 1-866-843-4681 for a recorded message. The hotline is updated daily when changing conditions affect bus operations during the late fall, winter, and early spring.

\section{Clinics}

MFC Dispensary 208-533-7210 Open Mon-Thur

$7 \mathrm{am}-5 \mathrm{pm}$

ATR Dispensary 208-533-4311 Open Mon-Thur $7 \mathrm{am}-5 \mathrm{pm}$

CFA Clinic

208-526-2356

Open 24-hours

SMC Dispensary

208-526-6263

Open Mon-Thur $7 a m-5 p m$

WCB Clinic

208-526-1596

Open Mon-Thur 7am-5:30pm 
Notes 
